\title{
Intervención de salud mental en niños expuestos a desastre natural
}

\author{
KATERINA SOMMER A. ${ }^{1}$, MARCELA ABUFHELE M. ${ }^{1}$, ANA MARINA BRICEÑO A. ${ }^{1}$, \\ ANAMARÍA DÁVILA D. ${ }^{2}$, MICHELLE BARREAU V. ${ }^{1}$, SONIA CASTRO M. ${ }^{2}$, \\ CAROLINA EBEL F. ${ }^{2}$, SANDRA OLTRA H. ${ }^{2}$, ALFONSO CORREA D. ${ }^{1}$
}

1. Psiquiatra Infantil y de la Adolescencia, Unidad Psiquiatría Infantil-Departamento de Pediatría-Clínica Alemana de Santiago, Facultad de Medicina Clínica Alemana- Universidad del Desarrollo, Santiago, Chile.

2. Psicóloga, Unidad Psiquiatría Infantil- Departamento de Pediatría-Clínica Alemana de Santiago, Facultad de Medicina Clínica Alemana-Universidad del Desarrollo, Santiago, Chile.

\begin{abstract}
Mental health intervention in children exposed to natural disasters

Introduction: This study is part of the mental health intervention, conducted by a child psychiatry team for children exposed to the February 2010 earthquake/tsunami in a community of the VII Region that was strongly affected by the natural disaster. Objective: To describe the intervention and evaluate the effectiveness of the strategies implemented for both children and teachers. Methodology: Interventions are described and classified in three categories (1) Case report and child care consulting, referred by their teacher. (2) Psychoeducational workshops for teachers of the intervened school. (3) Self-Care Day aimed at professionals of the same school. The evaluation is done through an anonymous survey designed to measure the effectiveness of the intervention. Results: 33 children were evaluated and treated, the most common diagnoses were adaptive disorders (8/33) and ADHD (11/33), and only 3 patients met the criteria for post-traumatic stress disorder (PTSD). Intervention implementation included psychoeducation for parents $(100 \%)$, coordination with schools and local health network (100\%), counseling (70\%) and drug prescription (45\%). Only $45 \%$ of the cases evaluated had symptoms triggered or exacerbated by the earthquake/tsunami. Regarding teacher evaluation $(\mathrm{N}: 11)$, $100 \%$ described the intervention as "very good". $90 \%$ considered it appropriate to the needs at the moment and as a contribution to their educational work. Conclusions: After events of this nature, many interventions take place to support the affected population. It is important to have more scientific information about the effectiveness of such interventions to prevent the development of post-traumatic psychopathology.
\end{abstract}

(Key words: Earthquake, tsunami, mental health, intervention).

Rev Chil Pediatr 2013; 84 (1): 59-67

Recibido el 14 de noviembre de 2012, devuelto para corregir el 22 de diciembre de 2012, aceptado para publicación el 07 de enero de 2013.

Este trabajo cumple con los requisitos sobre consentimiento/asentimiento informado, comité de ética, financiamiento, estudios animales y sobre la ausencia de conflictos de intereses según corresponda.

Correspondencia a:

Dra. Katerina Sommer Alarcón

katerinasommer@gmail.com 


\section{RESUMEN}

Introducción: Este trabajo forma parte de la intervención de salud mental, realizada por un equipo de psiquiatría infantil para niños expuestos al terremoto/maremoto de Febrero de 2010, en una comunidad de la VII Región fuertemente afectada por el desastre natural. Objetivos: Describir la intervención realizada y evaluar la efectividad de las estrategias implementadas tanto a niños como a profesores. Metodología: Se describe las intervenciones realizadas, clasificadas en 3 categorías: 1) Consultoría de casos clínicos y atención de niños derivados por sus profesores. 2) Talleres psicoeducativos a profesores de la escuela intervenida. 3) Jornada de autocuidado, dirigida a los profesionales de la misma escuela. La evaluación se realiza a través de encuesta anónima a los profesores diseñada para cuantificar la efectividad de la intervención. Resultados: Se evaluaron y trataron 33 niños, los diagnósticos más frecuentes fueron Trastornos adaptativos (8/33) y Déficit atencional (11/33); sólo 3 casos cumplían criterios de Trastorno por Estrés Postraumático(TEPT). Las intervenciones utilizadas incluyeron psicoeducación a padres $(100 \%)$, coordinación con colegios y red de salud municipal (100\%), apoyo psicológico (70\%) y farmacológico (45\%). Sólo en el $45 \%$ de los casos evaluados la sintomatología se había desencadenado o agravado con el terremoto/maremoto. En relación a la evaluación de profesores (n: 11), 100\% consideró la intervención como "muy buena". Un 90\% la consideró adecuada a las necesidades y constituyó un aporte a su quehacer educativo. Conclusiones: Tras eventos como éste, se realizan variadas intervenciones de apoyo a la población afectada. Es importante contar con mayor información científica acerca de la efectividad de dichas intervenciones para prevenir el desarrollo de psicopatología postraumática.

(Palabras clave: Terremoto, maremoto, salud mental, intervención).

Rev Chil Pediatr 2013; 84 (1): 59-67

\section{Introducción}

La mayoría de las reacciones que se pueden observar en los niños y adolescentes expuestos a un desastre constituyen respuestas esperadas; es decir, aun cuando son manifestaciones intensas de sufrimiento psicológico y pueden requerir de apoyo psicosocial, usualmente no son patológicas y la gran mayoría se resuelve en períodos cortos ${ }^{1}$. Sin embargo, la falta de información de los padres y las familias, la ausencia de comprensión por parte de los maestros y la confusión a la que se enfrentan los mismos niños, pueden generar que algunas de estas reacciones se compliquen tempranamente y generen secuelas ${ }^{2}$.

Está ampliamente descrito en la literatura, que a consecuencia de eventos traumáticos, tales como el terremoto sucedido el 27 de Febrero de 2010 en Chile, se pueden desencadenar trastornos psiquiátricos o el agravamiento de patología preexistente ${ }^{3-5}$, pudiendo manifestarse como alteraciones del ánimo, del sueño, de la conducta o cuadros ansiosos, como Trastornos de pánico, Fobias específicas y Trastorno por Estrés postraumático $(\mathrm{TEPT})^{3-6}$. Dentro de los cuadros mencionados, el TEPT es el de mayor severidad, pues en los niños puede provocar deterioro en áreas importantes de su desarrollo psicosocial y aumentar el riesgo de presentar psicopatología a futuro ${ }^{7-10}$.

Por otro lado, la evidencia plantea que el modo de prevenir la aparición de psicopatología grave en la población pediátrica, guarda relación con la forma cómo los niños y sus familias afrontan los eventos traumáticos y cómo se adaptan a las situaciones extremas. Esto depende de la edad del niño, de su posibilidad de comprensión de los eventos vitales a los cuales ha estado sometido, de la percepción de protección y de la seguridad que el entorno familiar le puede otorgar, junto con la precocidad en la contención y reorganización de la red de apoyo social ${ }^{1-3,11}$. La diferencia existente, por ejemplo, entre la frecuencia de TEPT en niños según la presencia o no de sintomatología ansiosa en los padres ha sido estudiada, concluyéndose que aumenta el riesgo de TEPT en niños cuyos padres están sintomáticos ${ }^{10}$.

Por estos motivos, ante desastres naturales altamente destructivos, se hace necesaria una intervención de salud mental integral, diseña- 
da específicamente para este grupo etario y en la que se contemplen acciones para los distintos miembros de la familia y de la comunidad afectada.

El presente trabajo describe la implementación final y la evaluación del programa de intervenciones de salud mental realizadas con los niños y profesores de una comunidad expuesta al desastre post terremoto y tsunami de Febrero del 2010. Se enmarca en el contexto de un artículo previo donde se describe cómo se gestó esta intervención y los pasos llevados a cabo para el diseño y coordinación de ésta ${ }^{1}$.

Esta experiencia fue diseñada y realizada en el marco del convenio de colaboración con la Municipalidad correspondiente, con el objetivo de minimizar las consecuencias negativas sobre la Salud Mental de la población afectada secundarias a la situación de crisis, promoviendo una mejor adaptación a la nueva reorganización de la comunidad afectada. Como objetivos específicos se planteó, a nivel de los niños, realizar evaluación, tratamiento y seguimiento de aquellos niños, derivados por sus profesores, que presentaban sintomatología emocional, conductual y/o somática, que interfiriese con su desarrollo o funcionamiento social regular, no necesariamente relacionado con el terremoto. A nivel de los profesores se buscó entregar estrategias de apoyo con el fin de fortalecer sus potencialidades individuales, como figuras importantes de contención, protección y pesquisa de niños sintomáticos; desarrollar herramientas de autocuidado grupales y sociales que favorezcan habilidades de expresión de emociones y resolución de conflictos, promoviendo la valoración personal y del otro y la sensación de ser competentes en sus labores profesionales.

\section{Participantes y Método}

\section{Diseño de la intervención}

Previo al diseño de la intervención, se realizaron visitas a la zona afectada para poder realizar un diagnóstico, con el fin de evaluar las necesidades específicas de los niños, sus padres y profesores, que se describe en detalle en un artículo previo del equipo ${ }^{1}$. En esta in- tervención se planteó hacer foco en los niños, en sus familias y en los profesores, desde la realidad de la escuela y, a su vez, validando la acción del servicio de atención de urgencia, donde se realizaron otras intervenciones de salud complementarias. Se escogió como foco la escuela, debido a que la consulta espontánea por salud mental infantil en el dispositivo de salud correspondiente (Posta Rural) era de muy baja frecuencia. La programación se organizó recogiendo las sugerencias de los profesores y directivos de la escuela.

Para una acción más abarcadora, dada la importancia de prevenir patología en adultos expuestos a catástrofes y su gran influencia en el desarrollo de psicopatología en niños, se coordinó la atención con psiquiatras y psicólogos de adultos.

\section{Implementación de la intervención}

La intervención consistió en una visita a la escuela cada 15 días por al menos dos miembros del equipo: un psiquiatra y una psicóloga infanto-juvenil. Las visitas estaban calendarizadas con anticipación por la directora de la escuela. La intervención tuvo una duración de 8 meses.

Se realizaron 3 modalidades de intervenciones, clasificadas en:

1) Consultoría de casos clínicos y atención de niños derivados por sus profesores, con consentimiento de sus padres. En virtud desus peticiones, se solicitó a los profesores la derivación de los niños según su apreciación de dificultades, con hoja de interconsulta dirigida a los especialistas. En cada visita realizada por el equipo, se atendió un promedio de 8 niños con sus respectivos padres, dentro de los que se encontraban controles de atenciones realizadas en las visitas previas así como derivaciones nuevas. La atención individual tenía formato de consulta, donde se realizaron evaluaciones clínicas, psiquiátricas y psicológicas, diagnósticos, tratamiento farmacológico, intervenciones psicoterapéuticas de apoyo y psicoeducación a los padres. El apoyo farmacológico fue otorgado y mantenido por el equipo durante toda la intervención, según se requiriera.

Se realizaron en todas las visitas, reuniones del equipo con los profesores y directora de la 
escuela, para otorgar y recibir retroalimentación de los niños evaluados y entregarles herramientas para el manejo de ellos en la sala de clases.

2) Talleres psicoeducativos a profesores de la escuela en estrategias de contención, protección y manejo de niños expuestos al evento traumático, así como en capacitación frente a temas de salud mental infanto-juvenil, solicitados por el mismo cuerpo docente, con una modalidad expositiva-experiencial. Los temas discutidos fueron: Síndrome de déficit atencional, Expresión emocional, Manejo de la Agresión en la sala de clases y Capacitación en técnicas de relajación. El equipo docente enfatizó la necesidad de apoyo respecto a la nueva estructura de la escuela, en la que el número de alumnos por curso aumentó en forma considerable, ya que debió fusionar las escuelas de varias localidades destruidas por el desastre.

3) Jornada de autocuidado, dirigida a los profesionales de la escuela al finalizar laintervención, 9 meses después del desastre: A modo de cierre de proceso, se realizó una jornada de autocuidado para los profesores, donde se trabajó el tema del estrés laboral y fortalecimiento del equipo. Los objetivos centrales de la jornada fueron: promover acciones de autocuidado en los profesores, para tomar medidas eficientes de prevención y promoción de la salud, capacitándolos en identificar señales tempranas de estrés; identificar y reconocer las fortalezas y debilidades en el plano individual y del grupo que intervienen en la calidad de vida en el plano personal y laboral y reconocer e implementar recursos prácticos de manejo de las tensiones laborales.

Al término de la intervención, se realizó un plan de seguimiento y se coordinó la continuidad de las acciones realizadas con agentes locales de salud mental, a través del médico general, el psicólogo y auxiliares paramédicos de las tres postas rurales del sector, realizándose la derivación de cada caso al nivel de salud correspondiente.

\section{Método de evaluación de las intervenciones}

Las atenciones de salud mental individuales se evaluaron según criterios clínicos. Las intervenciones con profesores fueron evalua- das a través de una encuesta anónima autoaplicada (diseñada por el equipo de salud mental específicamente para la intervención realizada) a los 11 profesores de la escuela que participaron de la intervención, que se aplicó al cierre del proceso, es decir, 9 meses después del terremoto. La escala diseñada constaba de 6 ítems con respuestas tipo Likert. Se buscaba evaluar la efectividad percibida por los profesores de las 3 metodologías utilizadas (consultoría y atención de casos clínicos, talleres psicoeducativos y jornada de autocuidado).

\section{Resultados}

\section{Participantes}

Niños: Se evaluaron y trataron 33 niños. Del total, 21 eran varones y 12 mujeres, el promedio de edad fue de 9 años con un rango entre 3 y 16, concentrándose las derivaciones entre los 7 y 10 años (70\% de los niños).

Veintinueve niños fueron derivados al Equipo de Salud Mental por los profesores de la escuela, 3 fueron derivados por el psicólogo de la capital de la provincia y uno por médico general de la posta de la localidad. Se detectaron cuadros de salud mental en 6 padres, los que fueron derivados a psiquiatras de adulto, realizando la coordinación de estas atenciones, así como en cada visita se evaluó y reforzó el cumplimiento y adherencia de sus tratamientos.

Profesores: Participaron en los talleres psicoeducativos y en la jornada de autocuidado final un total de 11 profesores.

\section{Atención de casos individuales}

Los diagnósticos más frecuentes fueron: Síndrome por Déficit Atencional con Hiperactividad (11 casos/33\%) y Trastorno Adaptativo ( 8 casos/24\%); sólo 3 de los niños derivados cumplieron criterios de TEPT al momento de realizar la evaluación inicial. De los cuadros clínicos manejados por el equipo, un $27 \%$ se había desarrollado en relación con el desastre y un $18 \%$ de los niños presentó exacerbación de los síntomas de cuadros previos.

Las intervenciones utilizadas incluyeron psicoeduación a padres $(100 \%)$, coordinación con 
colegios y red de salud municipal (100\%), apoyo psicológico $(70 \%)$ y farmacológico $(45 \%)$.

En 3 casos se realizaron visitas domiciliarias cuando los niños no se presentaron al control que estaban citados y 2 casos requirieron la coordinación y trabajo en conjunto con servicio social de la Municipalidad. La evolución de todos los niños fue clínicamente satisfactoria y al finalizar la intervención se derivaron a sus respectivas redes de salud para mantener sus controles con especialistas.

En el contexto de un trabajo paralelo de los autores $^{12}$, se aplicó la escala CPSS para evaluar TEPT en 17 de los casos tratados (quienes tenían la edad adecuada para aplicar la escala). De éstos, sólo 2 casos obtuvieron puntajes sospechosos de TEPT (una niña con diagnóstico inicial de TEPT y un niño con diagnóstico inicial de Trastorno Depresivo). Los otros dos casos con diagnóstico inicial de TEPT puntuaron bajo el punto de corte al finalizar la intervención.

\section{Evaluación de las intervenciones}

La evaluación general de la intervención realizada por los profesores, tuvo un 100\% de respuestas en categoría "muy buena" (Tabla 1). Dentro de la evaluación cualitativa, los profesores consideraron que la intervención constituyó un gran aporte para la escuela y la comunidad. Valoraron que pudiera ser duradera en el tiempo (no sólo tras la crisis) y la calificaron como una buena instancia de diálogo entre el equipo de la escuela.

\subsection{Consultoría de casos}

Esta instancia tenía el objetivo de discutir en conjunto con los profesores los casos evaluados, esta situación enriqueció y amplió la visión del manejo de los niños.
Dentro de la evaluación realizada por los profesores a las distintas actividades realizadas para ellos, la mejor evaluada fue la discusión de casos individuales de alumnos con dificultades, por parte de los distintos profesionales a cargo de su tratamiento y educación, siendo esta instancia evaluada como "muy útil" por el $100 \%$ de los profesores encuestados.

Los profesores plantearon que el poder compartir con los profesionales de salud mental las visiones de síntomas y problemáticas presentes en estos niños, les otorgó la oportunidad de identificar herramientas para poder manejarlos en la sala de clases y empoderarse más en su rol, sintiéndose contenidos y validados en su visión y qué hacer.

El dedicar tiempo a hablar sobre estos casos, también permitió que los profesores aunaran criterios y plantearan modos de actuar más consistentes y fundamentados, sustentando un trabajo en equipo que permite contener como institución a estos niños más sintomáticos $\mathrm{y}$ conflictivos.

\subsection{Talleres psicoeducativos}

Los profesores plantearon dudas y solicitaron herramientas para el manejo de algunas situaciones específicas que dificultaban la realización de las actividades escolares. Dentro de este contexto se realizaron talleres sobre temas identificados por ellos mismos.

En relación a la evaluación cualitativa, los profesores solicitan que pueda repetirse el año siguiente, enfatizando en que los talleres entregaron información de alta relevancia para su labor educativa.

El taller evaluado como de mayor utilidad fue el de capacitación en técnicas de relajación, donde el 91\% de los profesores lo clasificó como útil y muy útil.

Tabla 1. Encuesta de evaluación de las intervenciones realizadas ( $n=11$ profesores)

\begin{tabular}{|lcccc|}
\hline Variables evaluadas & Muy bueno & Bueno & Regular & Malo \\
\hline Aporte a quehacer profesional & $91 \%$ & $9 \%$ & $0 \%$ & $0 \%$ \\
\hline Adecuación a necesidades & $91 \%$ & $9 \%$ & $0 \%$ & $0 \%$ \\
\hline Elección de temas de talleres & $55 \%$ & $45 \%$ & $0 \%$ & $0 \%$ \\
\hline Disponibilidad del equipo & $91 \%$ & $9 \%$ & $0 \%$ & $0 \%$ \\
\hline Evaluación general de las intervenciones & $100 \%$ & $0 \%$ & $0 \%$ & $0 \%$ \\
\hline
\end{tabular}


Además los profesores refirieron que el hecho de tomarse tiempo para hablar sobre ellos y las dificultades en la sala de clases, era una instancia enriquecedora, que les aportaba en lograr empatizar con los otros y mejorar el clima laboral, potenciando el trabajo en equipo. Los profesores pudieron identificar en ellos la necesidad de tales instancias y de buscar activamente espacios de autocuidado.

\subsection{Jornada de autocuidado}

En términos generales la jornada fue evaluada en un $90 \%$ de los profesores como muy buena, el 100\% lo reportó como un muy buen aporte para su vida personal y el $85 \%$ como un muy buen aporte para su quehacer profesional.

De la evaluación cualitativa, los profesores reportaron la necesidad de contar con actividades de autocuidado en forma sistemática, dadas sus condiciones laborales y particularmente la intervención a nivel de clima laboral en los equipos de trabajo docente.

Los profesores reconocieron en un $100 \%$ la utilidad de la intervención realizada con los niños, tanto como para el desempeño y funcionamiento de los alumnos, como para la labor docente dentro del colegio. Describieron que los niños disminuyeron su inquietud y conductas disruptivas, lo que además se vio reflejado en su rendimiento escolar.

\section{Evaluacion global de la intervención}

La evaluación general de la intervención realizada por los profesores, tuvo un $100 \%$ de respuestas en categoría "muy buena" (tabla 1).

Dentro de la evaluación cualitativa, los profesores consideraron que la intervención constituyó un gran aporte para la escuela y la comunidad. Valoraron que pudiera ser duradera en el tiempo (no sólo tras la crisis) y la calificaron como una buena instancia de diálogo entre el equipo de la escuela.

Tanto los profesores como los padres, destacaron cambios conductuales secundarios a la intervención, como la presencia de niños más tranquilos y menos agresivos en la sala de clases y sus hogares, así como familias más motivadas y comprometidas con la escuela. Entre los cambios emocionales detectados en los niños durante las sesiones de control, se observó clínicamente una disminución significativa de la angustia y sufrimiento y mejoría en el rendimiento académico y en la percepción de alegría.

El equipo de salud mental, evaluó satisfactoriamente la intervención realizada durante estos meses, pues sin ser un grupo de salud mental comunitaria, logró implementar y llevar a cabo una intervención sostenida en el tiempo. Así mismo, se evaluó positivamente el impacto que la intervención tuvo en los niños que se trataron, pues el motivo de derivación se resolvió en la totalidad de los casos.

\section{Discusión}

Para conocer experiencias previas sobre medidas implementadas frente a situaciones de catástrofes anteriores en nuestro país, se revisaron en detalle las intervenciones realizadas después del terremoto de Mayo de 1960 en el sur de Chile ${ }^{13-15}$. Llaman la atención las medidas de apoyo implementadas en la población pediátrica, las cuales fueron casi exclusivamente dirigidas al bienestar físico; en ese sentido es importante destacar el cómo ha ido evolucionando el enfoque hacia los niños, encontrándose en la actualidad los equipos más atentos a sus necesidades de manera integral, considerando como relevantes los factores emocionales y realizando intervenciones de prevención específicas para este grupo poblacional.

Las situaciones de emergencia y desastres son eventos que no se pueden predecir, por lo que las intervenciones que se realizan a continuación tienden a ser rápidas y planificadas en corto tiempo. Es importante enfatizar en la necesidad de contemplar un apoyo desde la salud mental en forma inmediata, posterior a eventos traumáticos como lo sucedido en el terremoto/ maremoto del el 27 de Febrero del 2010. Esto requiere el concebir con anticipación intervenciones para los efectos que producen estos fenómenos a mediano y largo plazo en las comunidades afectadas, así como en la población general $^{3,16-18}$. En orden de poder satisfacer esta demanda, los equipos de salud mental requieren estar preparados para enfrentar el desafío de entregar e implementar intervenciones 
concretas, informadas e idealmente validadas científicamente.

Se pudo observar en terreno que en este desastre en particular, la población de las localidades afectadas fue intensamente intervenida, principalmente durante las primeras semanas de ocurrido el desastre, pero de manera poco sistematizada y con escasa continuidad en el tiempo ${ }^{19}$. Esta ayuda tiende a tener un carácter superficial y por lo general está realizada por pequeños grupos de trabajo, movilizados por iniciativas individuales y habitualmente carecen de procedimientos estandarizados, por lo que cobra real importancia el evaluar la manera en que estas situaciones se han abordado en nuestro país, así como en otros lugares del mundo para poder generar pautas de acción adecuadas. Existe mucha evidencia sobre la mayor efectividad que posee el implementar intervenciones organizadas y diseñadas antes de ocurrida una catástrofe ${ }^{17-19}$.

Frente a desastres, uno de los factores fuertemente asociados a la efectividad de una intervención, es que las acciones de salud a realizar estén fuertemente coordinadas con los agentes locales. Se ha constatado en diversos tipos de catástrofes, que una de las tareas más importantes, y difíciles de lograr, es el liderazgo y la coordinación de la ayuda ${ }^{19,20}$.

De acuerdo con la Inter-Agency Standing Comité (IASC) ${ }^{22}$, en situaciones de desastre es necesario establecer un único grupo coordinador de la asistencia que integre las diversas iniciativas de apoyo, que potencie a la propia comunidad afectada a fin de que ésta retenga el control de la situación, participando en la toma de decisiones para ajustar los planes de ayuda a sus necesidades y facilitar la sustentabilidad en el tiempo. A falta de un organismo único de coordinación, la gestión de la catástrofe tiende a ser más desordenada, siendo ineficiente el control y seguimiento de la misma.

En este contexto, se destaca la importancia de las gestiones de organización y análisis del contexto social realizadas previamente por el equipo en la presente intervención, lo que permitió evaluar la situación local y establecer una adecuada coordinación entre las diversas entidades. Se considera que con estas acciones se generó (en los agentes de salud local y en la población) credibilidad, confianza y percepción de pertinencia de las acciones implementadas.

La población no había tenido espacio a detenerse a reflexionar y buscar apoyo emocional, debido a la urgencia de actuar y salir adelante. Los profesores se encontraban lidiando con sus propios problemas, además de la urgencia de contener a los niños y adaptarse rápidamente al nuevo funcionamiento y reorganización escolar. La intervención les permitió desarrollar herramientas nuevas para abordar y apoyar a sus alumnos, así como aportaron en facilitar el trabajo en equipo y mejorar el clima laboral, según ellos reportaron en las evaluaciones.

Los temas en los que los profesores solicitaron ayuda, dos meses después del terremoto/ maremoto, se refieren a preocupaciones usuales de cualquier escuela (problemas conductuales, déficit atencional, situaciones de violencia, inquietud, desobediencia y dificultades en el aprendizaje), encontrándose en ese momento con dos posibilidades de interpretación: que se tratara de una conducta evitativa, con mayor o menor nivel de adaptabilidad, dada la necesidad de seguir funcionando, y otra opción era que efectivamente la situación de desastre haya estado parcialmente elaborada, acorde al tiempo transcurrido en ese proceso, con las emociones contenidas adecuadamente, y que lo que manifestaban era la necesidad de abordar las temáticas usuales de la escuela, que no se había realizado previamente por la escasa disponibilidad de profesionales especializados en la red de salud local. Como se describe a continuación, la dificultad de los profesores de detectar a los niños con sintomatología de TEPT y derivarlos, hace sospechar que se trataba de la primera opción (evitación).

Cabe destacar que dentro de los niños evaluados llamó significativamente la atención los escasos cuadros de TEPT que se encontraron como diagnóstico de ingreso dentro de los alumnos derivados, sobre todo al comparar con los resultados de la encuesta específica para detectar TEPT al final de la intervención que se describe en otro artículo de los autores $^{12}$ y evidenciar la alta prevalencia de este trastorno en la población escolar general 8 meses después del desastre $(40,4 \%)$. Esto po- 
dría explicarse porque, al ser las derivaciones realizadas por los profesores, ellos estarían mucho más atentos a la presencia de conductas externalizantes y disruptivas que afectan el funcionamiento habitual y la organización escolar, por lo que pueden no detectar fácilmente cuadros emocionales que no interfieren visiblemente en la conducta o rendimiento de los niños, lo que está descrito ampliamente en la literatura ${ }^{23}$. Este fenómeno es también es frecuentemente observado en la práctica clínica, donde las derivaciones escolares suelen ser principalmente por conductas disruptivas ${ }^{(24,25)}$.

Referente a este punto, se ha descrito que además las personas que sufren eventos traumáticos, suelen presentar cierto grado de dificultad para observar y detectar cuadros de salud mental en las personas que los rodean, sobre todo si se encuentran interferidos por algún trastorno psicopatológico en ese momento ${ }^{26-28}$. Esto podría explicar la razón de por qué a los profesores les resultó difícil reconocer en los niños síntomas de TEPT, en especial cuando estos síntomas no interferían en forma evidente en su funcionamiento escolar habitual.

Para implementar estrategias comunitarias de intervención en población infantil, es recomendable tener en cuenta la necesidad de realizar métodos de evaluación de la población general, mediante la aplicación de escalas de screening al inicio de las intervenciones. Esto permitiría hacer un mejor diagnóstico, al detectar con mayor eficacia la población sintomática, intervenir de manera más eficiente y oportuna y finalmente, evaluar la efectividad de la intervención efectuada. Además, se hace necesario aplicar técnicas de intervención en desastres con respaldo científico, dentro de las cuales destacan la Psicoterapia CognitivoConductual y la técnica Eye Movement Desensitization and Reprocessing (EMDR) ${ }^{17}$. Es importante recalcar que el analizar estas situaciones de desastres, también se debiera investigar los factores de resiliencia que le permiten a las personas salir fortalecidas de situaciones de crisis, que se han denominado indicadores de Crecimiento Postraumático ${ }^{29}$, ya que al identificarlos será posible potenciarlos, y así ir aumentando los factores protectores de la población.

\section{Referencias}

1.- Correa A, Abufhele M, Briceño AM et al: Intervención de salud mental para niños expuestos al desastre en la comunidad de Iloca: Experiencia de la Unidad de Psiquiatría Infantil de Clínica Alemana. Rev Chil Psiquiatr. Neurol Infanc Adolesc 2011; 22 (1): 92-101. Disponible en http://www.sopnia.com/boletines/Revista\%20SOPNIA\%202011-1.pdf

2.- Abufhele M, Arab E, Villar MJ, García R: Protocolo de intervención en crisis para niños y adolescentes víctimas del desastre. Rev Chil Psiquiatr Neurol Infanc Adolesc 2010; 21 (1): 63-78. Disponible en http://www.sopnia. com/boletines/Revista\%20SOPNIA\%202010-1.pdf

3.- $\operatorname{Kar} N$ : Psychological impact of disasters on children: review of assessment and interventions. World Journal of Pediatrics 2009, 5 (1): 5-11.

4.- Goenjian A, Molina L, Steinberg AM: Posttraumatic stress and depressive reactions among Nicaraguan adolescents after hurricane Mitch. Am J Psychiatry 2001; 158: 788-94.

5.- Dogan-Ates A: Developmental differences in children's and adolescents' post-disaster reactions. Biol Psychiatry 2002; 51 (7): 519-31.

6.- American Psychiatric Association: Manual Diagnóstico y Estadístico de los Trastornos Mentales, DSM-IV TR. 2003. Washington; Ed. Masson.

7.- Bustos P, Rincón P, Aedo J: Validación Preliminar de la Escala Infantil de Síntomas del Trastorno de Estrés Postraumático (Child PTSD Symptom Scale, CPSS) en Niños/as y Adolescentes Víctimas de Violencia Sexual. Psykhe 2009; 18 (2): 113-26.

8.- Alisic E, Kleber R: Measuring Posttraumatic stress reactions in children: A preliminary validation of the children`s responses to trauma inventory. J Child Adolesc Trauma 2010; 3: 192-204.

9.- Bisson JI: Post-traumatic Stress Disorder. BMJ 2007; 334: 789-93.

10.- Li X, Huang X, Tan H, Liu A, Zhou J, Yang T: A study on the relationship between posttraumatic stress disorder in flood victim parents and children in Hunan, China. Aust N Z J Psychiatry 2010; 44(6): 543-50.

11.- Gewirtz A, Forgatch M, Wieling E: Parenting practices as potential mechanisms for child adjustment following mass trauma. J Marital Fam Ther 2008; 34 (2): 177-92.

12.- Briceño AM, Abufhele M, Dávila A, et al: Trastorno por Estrés Postraumático en niños de una localidad costera a 8 meses del terremoto/maremoto. Rev Chil Pediatr (en prensa).

13.- Rodríguez S, Sánchez V, Onetto B: Salud Mental en 
situaciones de desastres. Un primer estudio bibliográfico y crítico. En Sextas Jornadas de la Sociedad Chilena de Salubridad, Santiago de Chile, Mayo 1960. Pág. 119-33.

14.- Sánchez V, Barilari F, García A, Sapiaín J: Consideraciones para el manejo de los problemas creados por las catástrofes sísmicas del sur de Chile (1960). Sugerencias de la cátedra de psiquiatría al servicio nacional de salud. En Sextas Jornadas de la Sociedad Chilena de Salubridad, Santiago de Chile, Mayo 1960, pág. 139-49.

15.- Comisión Médico-Social del Servicio Nacional de Salud y Consejo de Defensa del Niño: Informe general sobre colocación de niños damnificados trasladados de la zona Sur a la provincia de Santiago. En Sextas Jornadas de la Sociedad Chilena de Salubridad, Santiago de Chile, Mayo 1960, pág. 159-61.

16.- García F, Mardones R: Prevención de trastorno de estrés post-traumático en supervivientes del terremoto de Chile de febrero de 2010: Una propuesta de intervención narrativa. Terapia Psicológica 2010; 28: 85-93.

17.- Marín C, López W: Atención psicológica post-desastres: más que un "guarde la calma". Una revisión de los modelos de las estrategias de intervención. Terapia Psicológica 2010; 28 (2): 155-60.

18.- Figueroa R, Marín H, González M: Apoyo psicológico en desastres: Propuesta de un modelo de atención basado en revisiones sistemáticas y metaanálisis. Rev Med Chile 2010; 138 (2): 143-51.

19.- Méndez M, Leiva M: Mapa exploratorio de Intervenciones Psicosociales frente al Terremoto del 27 de Febrero de 2010 en la zona Centro-Sur de Chile. Terapia Psicológica 2010; 28 (2): 193-202.

20.- Quarantelli E: Ten Criteria for Evaluating the Management of Community Disasters. Disasters 1997; 21: 39-56.

21.- Chen L, Liu Y, KC C: Integrated Community-Based
Disaster Management Program in Taiwan. A Case Study of Shang-An Village. Nat Hazards 2006; 37: 209-23.

22.- Comité Permanente entre Organismos (IASC) (2007): Guía del IASC sobre Salud Mental y Apoyo Psicosocial en Situaciones de Emergencia. Ginebra: IASC. Disponible en http://www.who.int/mental_health/emergencies/ iasc_guidelines_spanish.pdf

23.- De los Reyes A, Kazdin A: Informant Discrepancies in the Assessment of Childhood Psychopathology: A Critical Review, Theoretical Framework, and Recommendations for Further Study. Psychol Bull 2005; 131 (4): 483-509.

24.- Abikoff H, Courtney M, Pelham WE, Koplewicz H: Teachers' ratings of disruptive behaviors: The influence of halo effects. Journal Abnorm Child Psych 1993; 21 (5): 519-33

25.- MINSAL: Ministerio de Salud, Gobierno de Chile. Niñas, niños y adolescentes con trastorno hipercinetico/ de la atencion. Disponible en http://www.minsal.gob.cl/ portal/url/item/71e6235bf06c7770e04001011f017ac5. pdf. Accessed December 26, 2012.

26.- Jiménez A, Cubillos R: Estrés percibido y satisfacción laboral después del terremoto ocurrido el 27 de Febrero de 2010 en la zona centro-sur de Chile. Terapia Psicológica 2010; 28: 191-6.

27.- Fossion P, Rejas M: La transmisión transgeneracional de los traumas. Revista Redes: Revista de psicoterapia relacional e intervenciones sociales 2000; 7: 53-73.

28.- Maida AM, Molina ME, Basualto C, Bahamondes $C$, Loevendagar X, Abarca C: El abuso sexual de las madres. ¿Es un predictor del abuso sexual de los hijos? Rev Chil Pediatr 2005; 76: 41-7.

29.- Tedeschi RG, Calhoun LG: The Posttraumatic Growth Inventory: Measuring the positive legacy of trauma. J Trauma Stress 1996: 9; 455-71. 